\title{
EZcap: a novel wearable for real-time automated seizure detection from EEG signals
}

Article

Accepted Version

Olokodana, I. L., Mohanty, S. P., Kougianos, E. and Sherratt, S. R. ORCID: https://orcid.org/0000-0001-7899-4445 (2021) EZcap: a novel wearable for real-time automated seizure detection from EEG signals. IEEE Transactions on Consumer Electronics, 67 (2). pp. 166-175. ISSN 0098-3063 doi: https://doi.org/10.1109/TCE.2021.3079399 Available at https://centaur.reading.ac.uk/99181/

It is advisable to refer to the publisher's version if you intend to cite from the work. See Guidance on citing.

To link to this article DOI: http://dx.doi.org/10.1109/TCE.2021.3079399

Publisher: IEEE

All outputs in CentAUR are protected by Intellectual Property Rights law, including copyright law. Copyright and IPR is retained by the creators or other copyright holders. Terms and conditions for use of this material are defined in the End User Agreement. 


\section{CentAUR}

Central Archive at the University of Reading

Reading's research outputs online 


\title{
EZcap: A Novel Wearable for Real-Time Automated Seizure Detection from EEG Signals
}

\author{
Ibrahim L. Olokodana, Student Member, IEEE, Saraju P. Mohanty, Senior Member, IEEE, \\ Elias Kougianos, Senior Member, IEEE, R. Simon Sherratt, Fellow, IEEE
}

\begin{abstract}
Epileptic seizures present a serious danger to the lives of their victims, rendering them unconscious, lacking control, and may even result in death only a few seconds after onset. This gives rise to a crucial need for an effective seizure detection method that is fast, accurate, and has the potential for mass market adoption. Kriging methods have a good reputation for high accuracy in spatial prediction, hence, their extensive use in geostatistics. This paper demonstrates the successful application of Kriging methods for an effective seizure detection device in an edge computing environment by modeling the brain as a spatial panorama. We hereby propose a novel wearable for real-time automated seizure detection from EEG signals using three different types of Kriging, namely, Simple Kriging, Ordinary Kriging and Universal Kriging. After multiple experiments with electroencephalogram (EEG) signals obtained from seizure patients as well as those from their healthy counterparts, the results reveal that the three Kriging methods performed very well in accuracy, sensitivity and latency of detection. It was found however, that Simple Kriging outperforms the other Kriging methods with a mean seizure detection latency of 0.81 sec, a perfect specificity, an accuracy of $97.50 \%$ and a sensitivity of $94.74 \%$. The results in this paper compare well with other seizure detection models in the literature but their excellent seizure detection latency surpasses the performance of most existing works in seizure detection.
\end{abstract}

Index Terms-Smart Home, Smart Healthcare, Smart Wearable, Seizure Detection, Epilepsy, EEG Signal, Kriging Methods, Edge Computing

\section{INTRODUCTION}

Epilepsy is among the most common neurological disorders and it affects over 50 million people in the world without any regard for race, age or gender [1]. People suffering from epileptic seizures have higher mortality rate compared to the wider population [2]. Most causes of death in epilepsy, apart from the Sudden Unexpected Death in Epilepsy (SUDEP) [3], can be prevented by early and accurate seizure detection, immediately followed by quick intervention from caregivers.

Kriging methods have been used extensively in geostatistics for spatial prediction. They exploit the covariance which exists within the data points to determine an estimate with minimal error [4]. Although Kriging enhances the accurate performance of seizure detection model in this paper, the latency advantage

Ibrahim L. Olokodana is with the Dept. of Computer Science and Engineering, University of North Texas, USA, E-mail: IbrahimOlokodana@my.unt.edu.

Saraju P. Mohanty is with the Dept. of Computer Science and Engineering, University of North Texas, USA, E-mail: saraju.mohanty@unt.edu.

Elias Kougianos is with the Dept. of Electrical Engineering, University of North Texas, USA, E-mail: elias.kougianos@unt.edu.

R. Simon Sherratt is with the Department of Biomedical Engineering, University of Reading, UK, E-mail: r.s.sherratt@ reading.ac.uk. is obtained through edge computing. Edge computing reduces latency by placing computation and data source in close proximity, which reduces the travel time from the source of data to the processing area. This is why the EZcap in Fig. 1 is situated directly on the head of the seizure patient where the EEG signals to be examined come from.

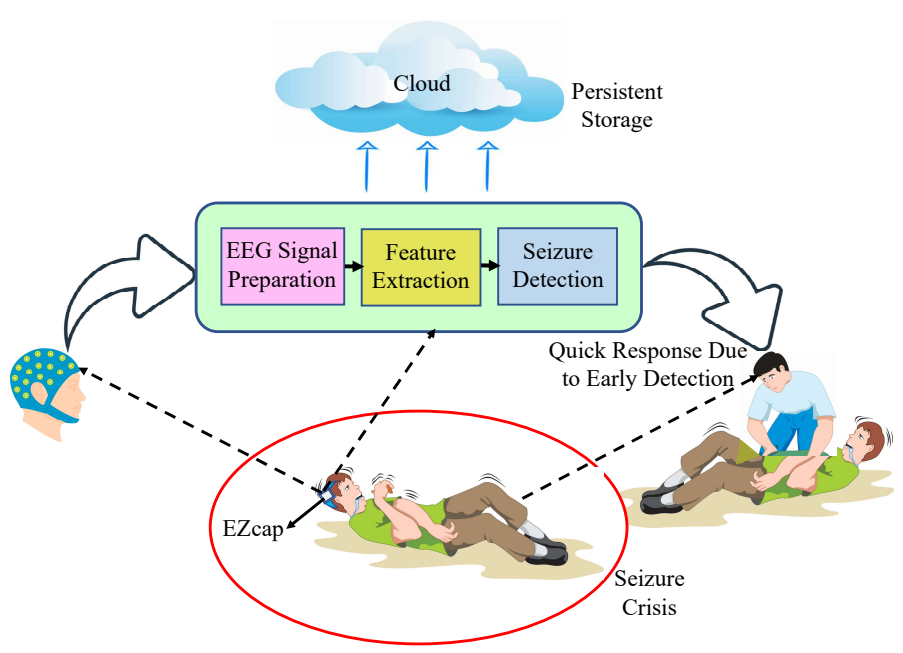

Fig. 1. Real-Time Seizure Detection for Rapid Response.

Fig. 1 depicts the merits of a timely seizure detection and the possible outcome of an undetected seizure or a late detection of seizure. The smart wearable EZcap on the subject's head is a portable device that will not hinder the patients' mobility so that seizure can be detected without restriction to a specific place or time. The EEG signal processing prior to seizure detection is carried out in the EZcap wearable that is situated right on the head of the patient, leading to a fast detection of seizure with low latency and a swift response by the assigned care-givers to prevent the victim from fatal injuries. The cloud shown in Fig. 1 is used as a means of data storage for use by a physician or research scientists since its capacity is larger, rather than its use for computation.

The rest of this paper is organized as follows: Section II is a review of related works in consumer electronics for smart healthcare. Section III discusses contributions of the current paper. Section IV describes a high level view of the proposed EZcap seizure detector. Section V presents the motivation for the choice of Kriging in seizure detection. Section VI is a brief discussion on Kriging methods and their theoretical perspective. Our proposed embedded logical architecture for the EZcap seizure detector is described in 
section VII. Experiments and results are stated in Section VIII while Section IX discusses the conclusion and future works.

\section{Related WORKS IN CONSUMER EleCtronics FOR SMART HEALTHCARE}

\section{A. Existing Consumer Electronics for Smart Healthcare}

Consumer electronics development for smart healthcare has received a big boost in the last decade. Proliferation of sensors, advancements in battery technology, availability of data,and increase in computational power have all contributed to the success. Table I summarizes the main categories of consumer electronics that have been proposed in the last ten years. The categories include fall detection, biosignal acquisition, stress and nutrition, disease monitoring, security and others.

A threshold system involving the use of a cardiotachometer and accelerometer for fall detection in senior citizens was proposed in [5]. The device was tested on 30 different subjects and recorded a decent accuracy and sensitivity of $97.50 \%$ and $96.80 \%$ respectively. A reduction in fall detection time by $38 \%$ is achieved by using a hybrid of electrocardiography (ECG) and the global positioning system (GPS) together with a proposed multi-thread method [6].

Smart acquisition of biosignals is an important aspect of smart healthcare and the Internet of Medical Things (IoMT). An Application Specific Integrated Circuit (ASIC)-based wireless biosignal acquisition consumer electronic device using Radio Frequency Identification (RFID) at an Ultra High Frequency (UHF) has been presented in [7]. A different approach involving the use of a smartwatch with local differential privacy to collect health data was proposed in [8].

Stress and nutrition are both related in the sense that stress can result in eating disorders. The combined effect of stress and improper nutrition can cause psychosomatic diseases. iLog was proposed as a consumer electronic device for providing information on an individual's emotional state with respect to his or her eating behavior [9], whereas Stress-Lysis detects the level of stress in a subject as proposed in [10].

Electrocardiography (ECG), Electroencephalography (EEG) and Photoplethysmography (PPG) have all been incorporated into consumer electronics for disease monitoring and management in recent years. An ECG-based consumer electronic device was proposed for the monitoring of arrhythmia disease in [11]. A detailed review of ECG-based cardiovascular disease monitoring was presented in [12]. A PPG-based approach for heart rate monitoring was proposed in [13], while EEG-based disease monitoring devices have been proposed in [14].

\section{B. Mass Market Consumer Devices for Seizure Detection}

Table II shows existing seizure detection devices suitable for mass market consumer use, and their basic features as compared to this current work.

Neuro-Detect [14] uses a combination of Hjorth Parameter features and a Deep Neural Network (DNN) in a hardwarein-the-loop consumer electronic design for seizure detection. EEG signals were first processed using the Discrete Wavelet Transformation (DWT) before features were extracted. While Neuro-Detect reported a decent performance, its use of Deep
Neural Network (DNN) which requires the use of many hyperparameters may result into high seizure detection latency.

eSeiz proposed the use of a signal rejection algorithm (SRA) by extracting hyper-synchronous pulses from EEG signals through a voltage level detector (VLD) [16]. The signal rejection algorithm works based on a threshold system in which signals below the threshold are rejected. A seizure is flagged if the amount of hyper-synchronous pulses exceeds the set threshold. The eSeiz consumer electronic proof of concept recorded a decent performance with very good power consumption. However, the reported seizure detection latency is more than 4 times the seizure detection latency in this current work. It also reported a specificity that is about $2.5 \%$ lower than what is recorded in this work. Furthermore, it is not clear how the eSeiz will respond to variations in signal complexities over time or a spontaneous surge in signal parameters since it uses a predetermined threshold value.

\section{Existing Research on EEG based Seizure Detection}

Many efforts on seizure detection in the literature focus on classification performance metrics like sensitivity, specificity and accuracy with limited interest on the computing environment [17]-[19]. A few researchers acknowledged the relevance of latency and added it as one of the metrics for measuring the performance of their proposed seizure detection model [16]. Machine learning algorithms are popularly used for the classification of seizure signals in many existing works, the most common being Artificial Neural Network (ANN) [18], Support Vector Machines (SVM) [17] and $\kappa$-Nearest Neighbor $(\kappa-\mathrm{NN})[20]$.

Other methods which are not based on EEG have been used as well for seizure detection. These methods employ the use of gyroscopes and accelerometers in wearable forms on the wrist or any other strategic part of the body to capture abnormal limb movement and unusual body vibrations during a seizure crisis [21], [22]. A major disadvantage of this approach is that normal activities such as dancing or sports could be confused for an epileptic vibration. Gaussian Process modeling was used in [23] for seizure detection in neonates but not in an edge computing paradigm.

In our previous work [24], Kriging methods have been explored for real-time seizure detection. However, in this extended version of our previous paper, a wearable design using Kriging methods for real-time seizure detection in an edge computing paradigm is proposed. This current work improves on the reliability and performance of our previous work by incorporating a hybrid system that comprises of both Kriging models and abnormal motion detection. In this case, seizure is only confirmed when the Kriging model flags a seizure and the motion sensor detects an abnormal body motion at the same time. Furthermore, in addition to the external escalation strategy, our new design also includes an alarm buzzer to create a local awareness that someone is in danger whenever there is a seizure attack. 
TABLE I

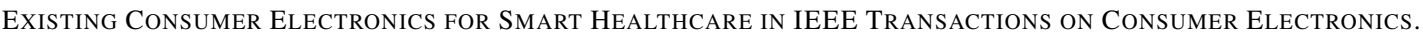

\begin{tabular}{|c|c|c|c|}
\hline Category & References & Implementation Method & Proposed Significance \\
\hline Fall Detection & [5], [6] & $\begin{array}{l}\text { Use of smart sensors such as the accelerometer, Global } \\
\text { Positioning System (GPS) and cardiotachometer to- } \\
\text { gether with a threshold system or multi-thread method } \\
\text { to segregate a fall incidence from a normal activity. }\end{array}$ & $\begin{array}{l}\text { To assist seniors who are generally vul- } \\
\text { nerable and susceptible to a fall get help } \\
\text { in time. }\end{array}$ \\
\hline Signal Acquisition & [7], [8] & $\begin{array}{l}\text { Design of an application specific integrated circuit } \\
\text { (ASIC) with radio frequency identification (RFID) at an } \\
\text { Ultra High Frequency (UHF) or the use of smartwatch } \\
\text { in an enhanced privacy environment achieved with local } \\
\text { differential privacy (LDP). }\end{array}$ & $\begin{array}{l}\text { Remote collection of bio signals for a } \\
\text { smart healthcare system. }\end{array}$ \\
\hline Health Monitoring & [11]-[13], [15] & $\begin{array}{l}\text { Use of Electrocardiography (ECG), Electroencephalog- } \\
\text { raphy (EEG) and Photoplethysmography (PPG) tech- } \\
\text { nologies to design consumer electronic products to } \\
\text { monitor and manage diseases such as arrhythmia, } \\
\text { epileptic seizures and so on. }\end{array}$ & $\begin{array}{l}\text { To prevent death or severe injuries } \\
\text { through early intervention and proper dis- } \\
\text { ease management. }\end{array}$ \\
\hline Stress and Nutrition & [9], [10] & $\begin{array}{l}\text { Design of consumer electronic products using multiple } \\
\text { sensors that are used to capure data that are analyzed } \\
\text { to estimate stress levels in a subject or predict the } \\
\text { nutritional value in a given meal. }\end{array}$ & $\begin{array}{l}\text { Stress level detection and nutritional bal- } \\
\text { ancing. }\end{array}$ \\
\hline
\end{tabular}

TABLE II

EXISTING CONSUMER Electronics FOR SEIZURE DETECTION.

\begin{tabular}{llll}
\hline $\begin{array}{l}\text { Existing } \\
\text { Work }\end{array}$ & Extracted Features & $\begin{array}{l}\text { Classification } \\
\text { Model }\end{array}$ & Latency \\
\hline $\begin{array}{l}\text { Neuro- } \\
\text { Detect [14] }\end{array}$ & Hjorth Parameters & $\begin{array}{l}\text { Deep Neural Net- } \\
\text { work (DNN) }\end{array}$ & $\begin{array}{l}\text { Not } \\
\text { reported }\end{array}$ \\
eSeiz [16] & $\begin{array}{l}\text { Hyper-synchronous } \\
\text { pulses }\end{array}$ & $\begin{array}{l}\text { Signal Rejection } \\
\text { Algorithm (SRA) }\end{array}$ & $\begin{array}{l}\text { Relatively } \\
\text { low latency }\end{array}$ \\
$\begin{array}{l}\text { Proposed } \\
\text { EZcap }\end{array}$ & $\begin{array}{l}\text { Fractal Dimension, } \\
\text { Entropy and Hjorth }\end{array}$ & 3D Kriging Mod- & $\begin{array}{l}\text { Very low la- } \\
\text { tency }\end{array}$ \\
\hline
\end{tabular}

\section{Novel Contributions of the CURRent PAPER}

\section{A. The Problem Addressed in the Current Paper}

Is it feasible to exploit the capacity of the brain to be represented as a spatial map in creating an effective seizure detection solution? In addition, assuming the brain can successfully take the form of a spatial map, will the success of Kriging in geostatistics be transferable to seizure detection? If the answer to this is yes, what Kriging method will be best suited for optimal performance in a smart wearable design using edge computing principles? The following sections of this paper address these research questions.

\section{B. The Challenges in Solving the Problem}

It is difficult to collect custom data-sets that will take full advantage of the properties of Kriging with respect to seizure detection because of the strict regulations in place for collecting human or animal data directly from the subjects. This leaves many researchers with the option of using publicly available data-sets which have been previously collected by qualified teaching hospitals [25]. The time complexity of conventional Kriging applications is also of concern but this was dealt with in this work by using only an already trained Kriging model on the edge device. Another challenge is the susceptibility of the collected EEG signals to noise. Security in the Internet of medical (IoMT) things needs to be addressed as well [26].

\section{The Solution Proposed in the Current Paper}

This paper proposes Kriging methods in a mobile and portable wearable design called EZcap for real-time seizure detection based on edge computing using the premise that the brain is capable of being modeled as a spatial map that is fitted for Kriging application. Three Kriging methods were explored in this work in order to identify the one that is most suitable for detecting seizure in real time, in an edge computing environment.

\section{The Novelty of the Solution Proposed}

Our specific modeling of the brain as a spatial representation that is suitable for Kriging application as it relates to seizure detection is novel. To the best of our knowledge, this is a pioneering work on the application of Kriging methods in a wearable configuration for real-time detection of epileptic seizures using edge computing design methodologies. The mean seizure detection latency reported in this work is less than one second, far surpassing the performance of many existing seizure detection models. Furthermore, our hybrid approach of combining abnormal motion detection and the empirical Kriging application to strengthen the effectiveness of our proposed seizure detector is novel, to the best of our knowledge.

\section{EZCAP: A Novel WeARABle For RAPID SEIZURE DETECTION AND NOTIFICATION IN EDGE COMPUTING}

EZcap is proposed as a light-weight consumer electronics device for early detection of seizure based on edge computing using a novel software-hardware interaction, as shown in Fig. 2. The hardware part of EZcap consists of an edge processing 
unit (EPU), motion sensor, buzzer and a display unit such as a liquid crystal display (LCD), while the battery and communication components are concealed under the hood. The software part consists of the EEG signal processing and feature extraction algorithms, as well as a Kriging model that has been trained to identify seizure signals.

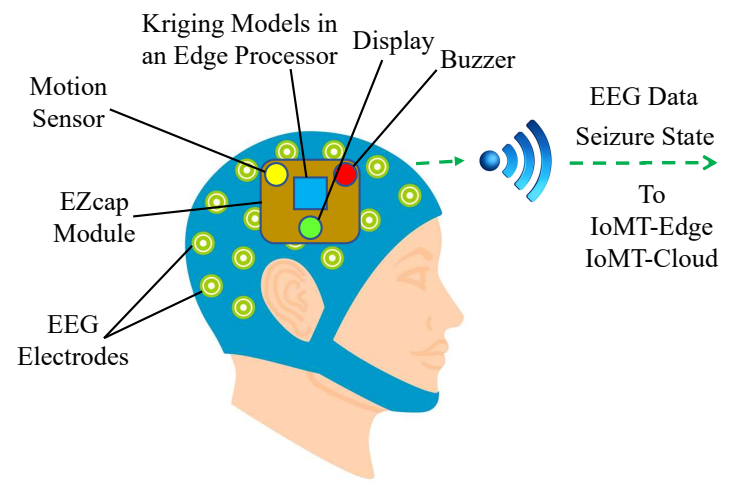

Fig. 2. Conceptual schematic of the proposed EZcap.

The motion sensor complements the EEG-based seizure detection using the Kriging model by sensing the abnormal movements of the body which are associated with a seizure attack. The buzzer acts as a local crisis alarm to alert persons in the closest radius of a patient in danger. The display unit shows the seizure status of the patient in real time and the power level of the device. Once a seizure is detected, apart from the local crisis alarm that is automatically activated, notifications are also forwarded to the physician and other caregivers in the vicinity of the patient. In addition, EEG data from the patient are consistently stored in the cloud for future patientspecific analysis. Seizure is only confirmed when the Kriging model detects a seizure and the motion sensor simultaneously senses an abnormal motion. This novel hybrid approach further strengthens the effectiveness of our proposed system. A user experience research conducted on the use of mobile EEG technologies shows a high acceptance rate after an initial discomfort in the first few minutes of wearing it [27]. This proves that the proposed EZcap has a high prospect as a mass market consumer device for seizure detection.

A detailed architecture of the EZcap seizure detector is illustrated in Fig. 3. The sensed EEG signals are processed locally (at the edge) by the EPU which determines the seizure state of the subject. Concurrently, all collected data along with their analysis are stored to the cloud for future reference as well as improved training. The EPU makes a determination on seizure state based on the EEG signals and the input of the motion sensor. Depending on the Kriging analysis and the input of the motion sensor, the system determines whether a seizure has occurred or whether there is suspicion of a seizure. In these cases, a buzzer sounds to alert caregivers and the LCD flashes a warning message. In all cases, the seizure state along with associated data is stored in the cloud.

Processing data at the edge is highly effective, mainly because more data are now manufactured at the user edge of the network than any other time in the past, owing to the multiplication of sensors and sensing mechanisms [28].

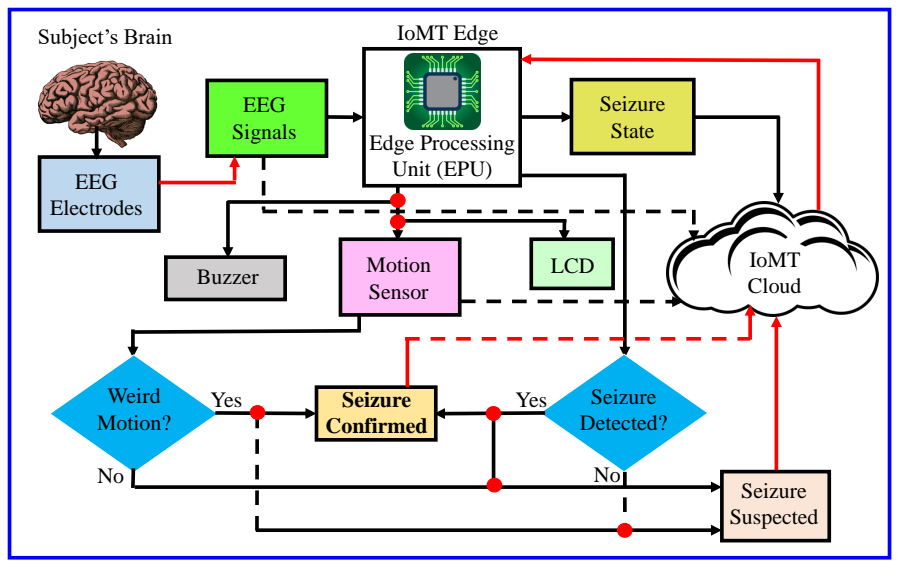

Fig. 3. Proposed architecture of EZcap seizure detector.

Mitigated deployment cost, reduced latency and portability are some of the merits resulting in the increasing acceptance of the edge computing paradigm. These merits are even more meaningful when life is threatened, as it is for an epileptic seizure situation. Apart from accuracy, three important features that are present in our proposed edge computing solution to seizure detection are portability, affordability and low latency. A seizure detection solution should be portable in order not to restrict the mobility of the patient to a confined space. It should be affordable to enable low-income areas of the world, who are the most impacted with the seizure disorder, access to the solution. It should also have low latency as this would help neutralize the threat to the patient's life during a seizure attack.

\section{KRIGING MOTIVATION - THE BRAIN ENVISIONED AS A SPATIAL MAP SUITABLE FOR SPATIAL DATA PROCESSING}

We conceptualize the brain as a spatial panorama that is ideal for the application of spatial data processing techniques such as Kriging for solving the seizure detection problem due to the following reasons:

1) The brain can be modeled as a multi-layered spatial entity with ceaseless dynamic activities [29]. It is further indicated that multiple similarities exist between the mapping of the brain and the geographical information system (GIS) mapping.

2) It has been discovered that some cellular activities in the hippocampal area of the brain generate maps for navigation and recognition [30]. It was stated in [25] that a part of the collected EEG readings from some patients of epilepsy were collected from the brain's hippocampus.

3) Kriging produces very good performance even on datasets that are relatively small compared to most machine learning methods which rely heavily on high data volume for a decent performance. This is important because of the challenges involved in collecting large amounts of biomedical datasets.

4) Kriging estimates are often accompanied by an estimation variance which is a measure of the credibility of 
the given estimate. There is an estimation variance for each Kriging estimate which clearly reveals whether the Kriging model worked well for that specific location or not.

5) Kriging models are very reliable and accurate despite not requiring many hyperparameters [?]. They are also robust to certain spontaneous events such as unexpected compromise in data quality or feature sets inconsistency [31].

Fig. 4 compares the spatial map of the brain (Fig. 4(b)) to a typical geographical map (Fig. 4(a)). Both are similar in terms of having multiple locations on which spatial continuity can be established or evaluated. Fig. 4(b) represents the map of the brain and its lobes which are comparable to geolocations of the earth. Kriging [32] is a geospatial technique popularly used for predictive estimation in spatial statistics where locations with known values of certain quantities are used to estimate the unknown values at the neighboring locations. The black and red circles shown in Fig. 4(b) represent the brain sites with unknown and known values of the considered variable, respectively. The broken lines are a measure of the spatial correlation within the sampled sites. Studies have shown that although seizures may emanate from a particular site in the brain, they can be spatially spread out to other areas of the brain [23]. Consequently, some locations with known variable values have seizures while others do not. It might therefore be feasible to estimate the specific locations of the brain that are seizure-inflicted. Nevertheless, the primary focus of this paper is to establish the effectiveness of Kriging models for seizure detection.

\section{Kriging Methods - A Theoretical Perspective}

The word "Kriging" was coined in honor of Daniel Krige who was instrumental to the development of geostatistical mining [33]. Kriging hinges on spatial continuity, a function of correlation over a given distance. This means that closer locations are better correlated than farther locations. Three major steps are involved in the Kriging process. First is the estimation of spatial continuity via the semi-variogram which shows variations in quantities with respect to distance, the second step is the model fitting on the semi-variogram generated in the first step while the final estimation using the fitted model is achieved in the third stage [34].

Fig. 5 shows various types of Kriging [33]. The three major types of Kringing, which are Simple Kriging, Ordinary Kriging, and Universal Kriging are the focus of this work.

\section{A. The Semi-variogram}

The semi-variogram is a scatter plot with points representing the average variation among a group of paired locations having the same separating distance that is called lag vector $\mathbf{h}$ [34]. The semi-variogram is generated according to the following formula:

$$
\gamma(\mathbf{h})=\frac{1}{2 \mathbf{N}(\mathbf{h})} \sum_{i=1}^{\mathbf{N}(\mathbf{h})}\left(\mathbf{Z}\left(\mathbf{x}_{i}\right)-\mathbf{Z}\left(\mathbf{x}_{i}+\mathbf{h}\right)\right)^{2}
$$

where $\gamma(\mathbf{h})$ is the semi-variogram at a specific lag vector $\mathbf{h}$ between a pair of points, $\mathbf{N}(\mathbf{h})$ represents the total count of lag vectors $\mathbf{h}$ for each point on the semi-variogram while $\mathbf{Z}\left(\mathbf{x}_{i}\right)$ represents a Gaussian process on the sampled observations $\mathbf{x}_{1}, \mathbf{x}_{2}, \ldots, \mathbf{x}_{i}$ at various locations.

\section{B. The Semi-variogram Model}

This is used to fit a curve on the semi-variogram scatter plot. Fig. 6 is a taxonomy of different types of semi-variogram models.

The selection of a semi-variogram model depends on the kind of spatial relationship existing in the semi-variogram scatter plot [34]. The Gaussian semi-variogram model was the favored choice for this work due to the underlying Gaussian properties of the recorded EEG time-series. The Gaussian semi-variogram model is expressed mathematically as follows:

$$
\gamma(\mathbf{h})=\left\{\begin{array}{cc}
C\left[1-\exp \left(-\frac{\mathbf{h}^{2}}{a^{2}}\right)\right] & \mathbf{h}>0 \\
0 & \mathbf{h}=0
\end{array}\right.
$$

In the above expression, $C$ represents the total variance contribution known as the sill and $a$ is the horizontal component of the sill known as the range.

\section{Kriging Estimate}

This is the final estimation at locations with unknown values using the semi-variogram model that was fitted earlier. Kriging places weights on the link between pair locations, proportional to their auto-correlation. Hence, it is sometimes called Best Linear Unbiased Estimator (BLUE) [35].

The three basic types of Kriging differ based on their assumption regarding the global mean $\left(\mu_{z}\right)$ for the intrinsic Gaussian process $\mathbf{Z}(\mathbf{x})$. Simple Kriging assumes a known constant mean, Ordinary Kriging assumes an unknown but constant global mean while Universal Kriging assumes a varying global mean [32].

The other types of Kriging (Fig. 5) have at least one of the above global mean assumptions.

The covariance $C(\mathbf{h})$ of a location pair is derived as follows from the semi-variogram model [4]:

$$
C(\mathbf{h})=C(0)-\gamma(\mathbf{h}),
$$

where $C(0)$ represents the sill.

Multiplying the covariance for each known location pair by specific weights results in a system of equations:

$$
\mathbf{C}_{n \times n} \cdot \boldsymbol{\lambda}_{n \times 1}=\mathbf{c}_{n \times 1},
$$

where $\mathbf{C}_{n \times n}$ is the covariance matrix for known location pairs, $\mathbf{c}_{n \times 1}$ represents a covariance vector of every point in relation to the unknown, $\boldsymbol{\lambda}_{n \times 1}$ represents a weight vector and $n$ is the total number of points.

We therefore have,

$$
\boldsymbol{\lambda}_{n \times 1}=\left(\mathbf{C}_{n \times n}\right)^{-1} \mathbf{c}_{n \times 1} .
$$




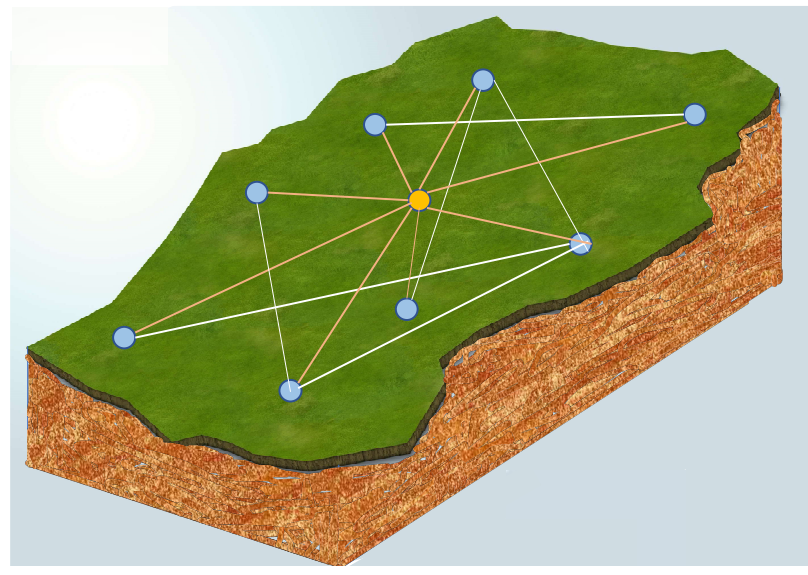

(a) A geographical map showing correlation among multiple locations.

Fig. 4. Schematic representation of the brain as a spatial map.

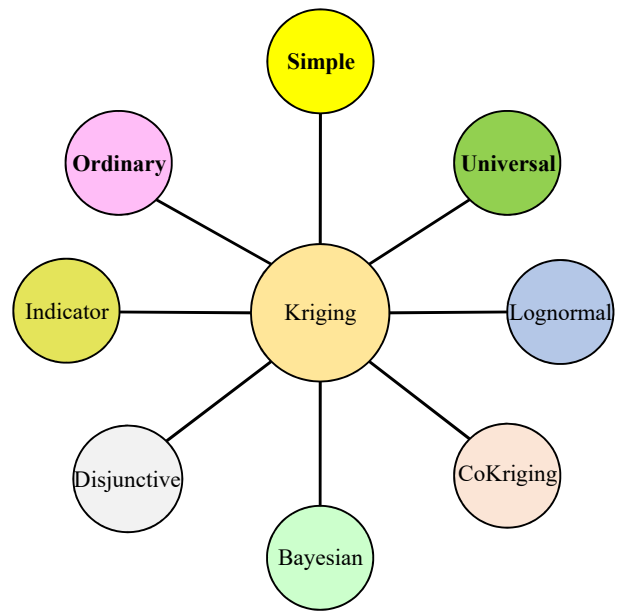

Fig. 5. The different types of Kriging methods.

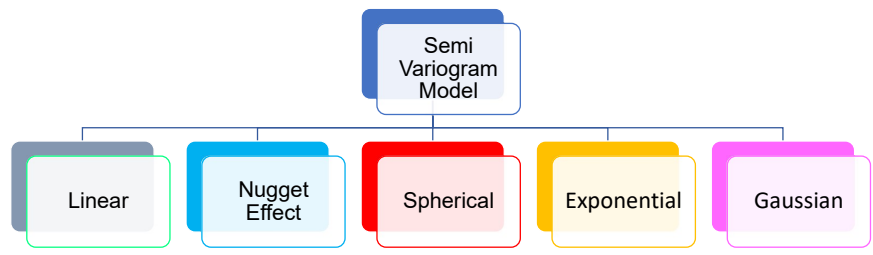

Fig. 6. Semi-variogram models

The Kriging estimate $y\left(\mathbf{x}_{o}\right)$ is then finally obtained by multiplying the weight vector (Eqn. 5) with the residual $\mathbf{R}\left(\mathbf{x}_{i}\right)$ as shown below:

$$
y\left(\mathbf{x}_{o}\right)=\sum_{i=1}^{n} \lambda_{i} \mathbf{R}\left(\mathbf{x}_{i}\right),
$$

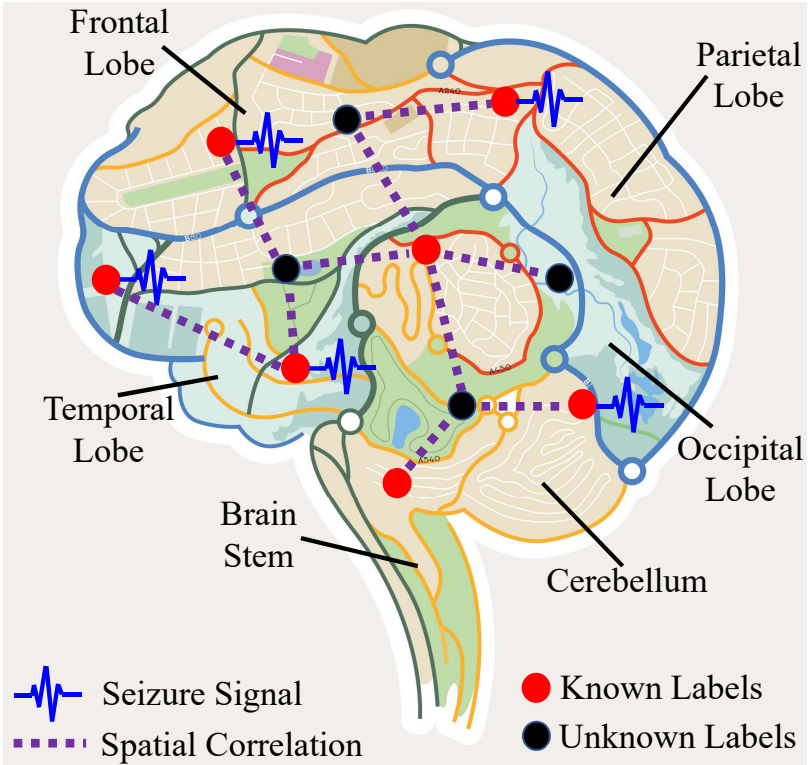

(b) Map of the brain showing seizure regions and spatial correlation.

where

$$
\mathbf{R}\left(\mathbf{x}_{i}\right)=\sum_{i=1}^{n}\left(Z\left(\mathbf{x}_{i}\right)-\mu_{z}\right)
$$

\section{Proposed Novel Kriging-Based Seizure Early DETECTOR}

The proposed EZcap seizure detector has a novel logical block that is situated inside the edge processing unit (EPU) shown in Fig. 2.

The proposed novel logical architecture of our Krigingbased early seizure detector as shown in Fig. 7 is comprised of the signal de-noising unit; the feature extraction block which consists of the Hjorth complexity unit, fractal dimension unit and the singular value decomposition (SVD) entropy unit; the variogram estimation unit; the Kriging estimation unit and the seizure status unit.

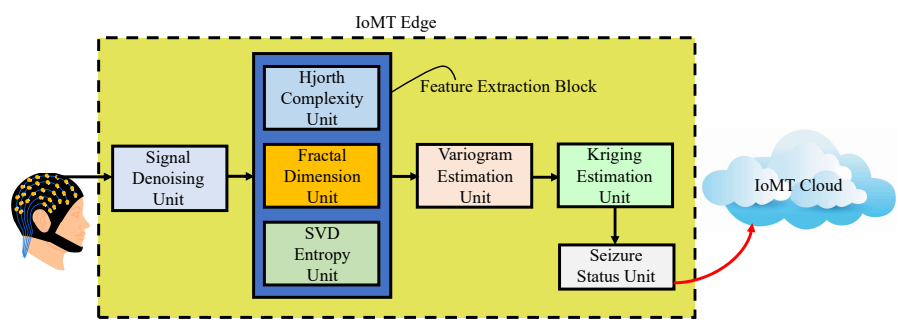

Fig. 7. Proposed embedded architecture of EZcap seizure detector.

The signal de-noising unit removes noise, especially due to artifacts and physiology, from the EEG signals collected from the subject using a discrete wavelet transform (DWT) soft thresholding technique. Three unique features are then extracted from the clean EEG signal in the feature extraction block. These features are processed in the Hjorth complexity unit, fractal dimension unit and the SVD entropy unit. Upon 
extraction of the features, the vectorized features are passed to the variogram estimation unit, which generates the semivariogram, and subsequently to the Kriging estimation unit for the final processing to obtain a seizure outcome. Further details on the semi-variogram and Kriging estimation are presented in Section VIII. The seizure status unit presents the subject's seizure state and relocates the outcome to the external IoMT cloud for further processing and storage. Activities in the IoMT cloud may include communication with emergency services, personal physician and family members or related individuals in order to trigger a prompt and effective rescue of the subject who might be suffering from an epileptic seizure crisis.

\section{EXPERIMENTAL RESULTS AND THEIR ANALYSIS}

\section{A. EEG Dataset and Extracted Features}

The dataset for this work [25] has been used for many seizure detection research projects [17], [20]. It consists of five sets A to E. Set A consists of healthy signals while set E comprises the seizure EEG signals. These are the two sets used in this work. Sets B, C and D are healthy and inter-ictal signals. Each set contains 100 EEG segments which were sampled at $173.61 \mathrm{~Hz}$.

Three features were extracted from the EEG signals. They are Fractal Dimension, Hjorth Complexity and Singular Value Decomposition (SVD) Entropy. While two of the features model the location coordinates, the other feature being Fractal Dimension models the quantity of interest that will be estimated. The mathematical expressions of the features are given in Eqns. 8 to 11 .

$$
\text { Fractal_Dimension }=\frac{\log _{e}(n)}{\log _{e}(n)+\log _{e}\left(\frac{n}{n+0.4 N_{\delta}}\right)},
$$

where $n$ is number of data points in the EEG sequence or simply length of the sequence and $N_{\delta}$ represents the number of alternating pair of signs in the inherent binary sequence.

$$
\text { Hjorth_Complexity }=\frac{\text { Mobility }\left(\frac{d x(t)}{d t}\right)}{\operatorname{Mobility}(x(t))} .
$$

In the above expression, Mobility is calculated as follows:

$$
\text { Mobility }=\sqrt{\frac{\operatorname{var}\left(\frac{d x(t)}{d t}\right)}{\operatorname{var}(x(t))}},
$$

In the above expression, $x(t)$ and $t$ are the voltage amplitude and corresponding time, respectively. Singular Value Decomposition (SVD) Entropy is calculated as:

$$
S V D \_ \text {Entropy }=\sum_{i=1}^{M} \bar{\sigma}_{i} \log _{2}\left(\bar{\sigma}_{i}\right),
$$

where $M$ is the number of singular values of an embedded matrix and $\bar{\sigma}_{1}, \bar{\sigma}_{2}, \ldots, \bar{\sigma}_{M}$ are the normalized singular values of the embedded matrix.

Fig. 8 shows the distribution of the EEG segments as data points using the extracted features. The figure roughly shows

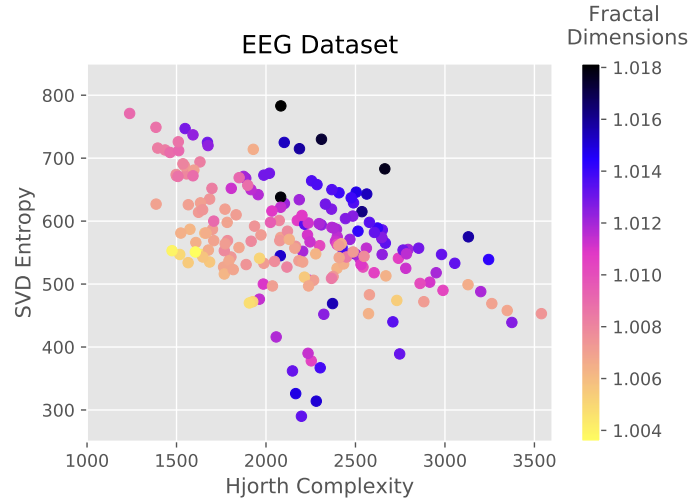

Fig. 8. Feature representation of EEG dataset using color map.

two categories through a simple observation of the colors of the data points except for a few outliers which may be attributed to some residual noise in the data-set. This reveals the spatial continuity in the data-set and proves the quality of the selected features. It also serves as an initial validation of our three-dimensional modeling of the seizure detection problem towards the application of Kriging.

\section{B. Training the Kriging Models}

The EEG segments in the data-set were randomly split into two, according to the $80 / 20$ rule [36]. The larger part is the training set while the other part is reserved for testing. The training follows the outlined process in section VI. We first obtain the semi-variogram of the training set using Eqn. 1 and then fit the semi-variogram using the Gaussian semi-variogram model in Eqn. 2. Fig. 9(a) and 9(b) are the semi-variogram plot and the fitted semi-variogram plot of the training set, respectively.

The covariance matrix used to generate the Kriging weights is extracted from the fitted semi-variogram. The Kriging estimates of the fractal dimensions are then finally calculated by multiplying the Kriging weights with the residuals (Eqn. 6). The resulting Kriging estimates are resolved to one of two states as either healthy (represented by "0") or ictal (represented by " 1 ").

\section{Performance Metrics for Edge Computing Paradigm based Seizure Detection}

After obtaining the seizure states from the Kriging estimates, the performance of the three Kriging methods under consideration were computed and compared based on accuracy, sensitivity, specificity, F1-score and latency metrics using edge computing principles. The models were trained on a workstation because of the third order time complexity of Kriging and then ported to an edge device for the actual realtime detection of seizure. Table III shows the performance comparison of the different Kriging methods.

From the presented results in Table III, Simple Kriging and Ordinary Kriging produced equal performance at $99.7 \%$ and 95.4\% Confidence Intervals (CI). However, Simple Kriging surpassed the performance of Ordinary Kriging at $68.2 \%$ 


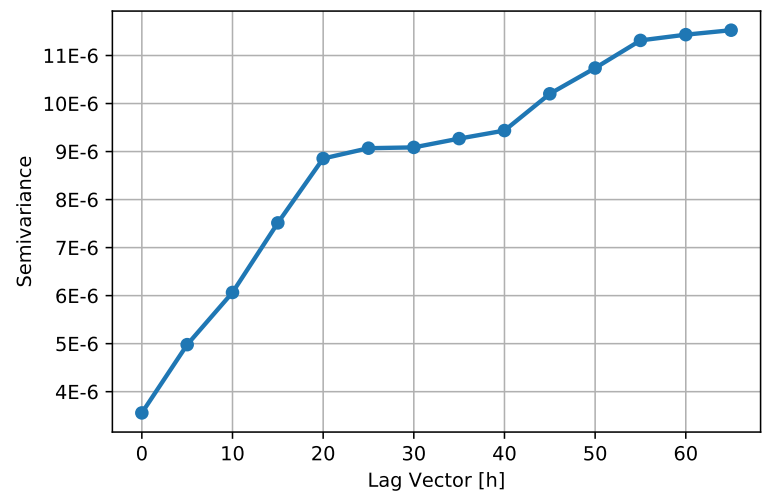

(a) Semi-variogram of the EEG training set.

Fig. 9. Modeling of the semi-variogram for Kriging prediction.

TABLE III

COMPARING KRIGING PERFORMANCES FOR SEIZURE DETECTION AT DIFFERENT CONFIDENCE INTERVALS (CI)

\begin{tabular}{llccc}
\hline \hline C. Int. (CI) & Kriging Models & Accuracy & Sensitivity & Specificity \\
\hline \multirow{2}{*}{$99.7 \%$ CI } & Simple Kriging & $97.50 \%$ & $94.74 \%$ & $100.00 \%$ \\
& Ordinary Kriging & $97.50 \%$ & $94.74 \%$ & $100.00 \%$ \\
& Universal Kriging & $80.00 \%$ & $89.47 \%$ & $71.43 \%$ \\
\hline \multirow{3}{*}{$95.4 \%$ CI I } & Simple Kriging & $92.50 \%$ & $94.74 \%$ & $90.48 \%$ \\
& Ordinary Kriging & $92.50 \%$ & $94.74 \%$ & $90.48 \%$ \\
& Universal Kriging & $80.00 \%$ & $89.47 \%$ & $71.43 \%$ \\
\hline \multirow{2}{*}{$68.2 \%$ CI I } & Simple Kriging & $90.00 \%$ & $89.47 \%$ & $90.48 \%$ \\
& Ordinary Kriging & $87.50 \%$ & $84.21 \%$ & $90.48 \%$ \\
& Universal Kriging & $80.00 \%$ & $89.47 \%$ & $71.43 \%$ \\
\hline
\end{tabular}

CI. Universal Kriging came behind across every confidence interval that was considered. This could be due to Universal Kriging's superior complexity compared to the other two methods, given the limited size of the dataset, which is common in biomedical signal processing.

Fig. 10 is a histogram plot of the F1 scores of the Kriging methods on the seizure detection task. It further corroborates the superiority of Simple Kriging method over others, especially at $68.2 \%$ CI with Ordinary Kriging as a close second.

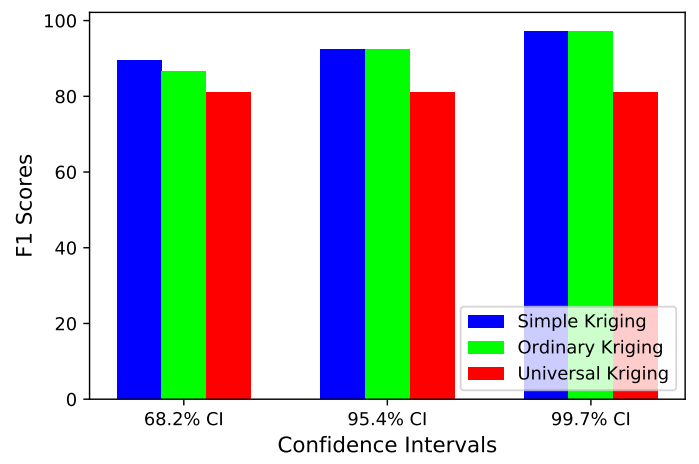

Fig. 10. Kriging performance comparison using F1-scores and confidence intervals.

Table IV lists the latency performances of the Kriging models on the edge hardware. Simple Kriging again has the lowest mean detection latency of $0.81 \mathrm{~s}$ while Universal Kriging has the highest latency of $16.25 \mathrm{~s}$. The mean detection

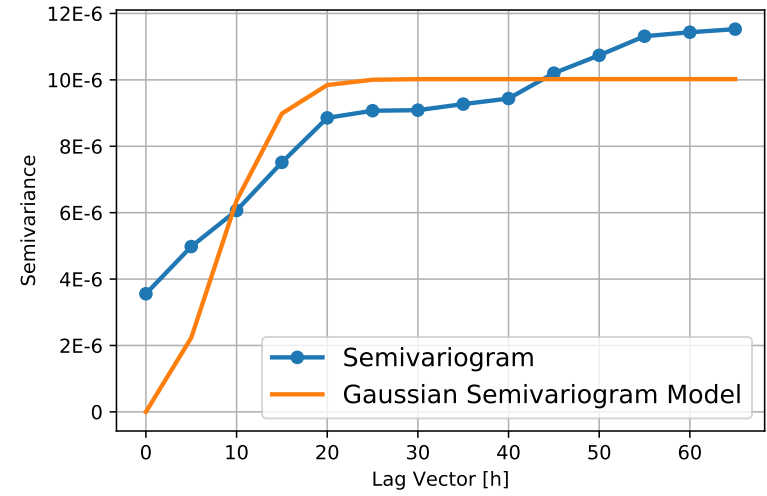

(b) Fitted semi-varigram using the Gaussian semivariogram model.

latency was calculated as an average of ten trials for each Kriging method.

TABLE IV

COMPARING MEAN DETECTION LATENCY OF KRIGING MODELS IN AN EDGE COMPUTING PARADIGM

\begin{tabular}{lc}
\hline \hline Kriging Models & Detection Latency (s) \\
\hline Simple Kriging & $\mathbf{0 . 8 1}$ \\
Ordinary Kriging & 0.86 \\
Universal Kriging & 16.25 \\
\hline
\end{tabular}

Table $\mathrm{V}$ shows the comparison of our proposed seizure detector to other existing works in the literature. The latency performance of our model is clearly superior to that of the other works in the literature with a marginal compromise in accuracy. However, the marginal lapse in accuracy is compensated by the presence of the motion sensor in our design. The motion sensor detects any abnormal body movement and triggers an alarm in case the model misses a seizure.

\section{CONCLUSION AND FutURE WORK}

The effectiveness of Kriging methods in a wearable design for real time seizure detection in an edge computing paradigms has been explored here by modeling the brain as a three-dimensional spatial representation that is similar to a geographical landscape suitable for the application of Kriging. Three Kriging models were evaluated across various metrics. Simple Kriging shows the best performance, Ordinary Kriging comes very close but Universal Kriging was quite far behind. In addition to the performance of our proposed seizure detector, the encouraging acceptance rate of mobile EEG technologies among users shows that EZcap is highly promising as a mass market consumer electronic device for seizure detection.

The work presented here has great potential of benefiting consumers with epilepsy in future consumer electronic devices. The authors intend to further explore the potentials of Simple Kriging for more efficient and time-sensitive seizure detection and prediction wearable designs. Incorporation of drug-delivery mechanisms into the seizure detector to provide seizure control and early detection in a unified system in IoMT is a future research [41]. We also plan to explore 
TABLE V

COMPARING RESULTS WITH EXISTING WORKS IN THE LITERATURE.

\begin{tabular}{|c|c|c|c|c|c|c|}
\hline Published Works & Extracted Features & Classification Algorithm & Accuracy & Sensitivity & Specificity & Detection Latency \\
\hline Shoeb, et al. [37] & $\begin{array}{l}\text { Spectral, temporal and } \\
\text { spatial features. }\end{array}$ & $\begin{array}{l}\text { Support Vector Machine } \\
\text { (SVM) }\end{array}$ & NA & $96.00 \%$ & 0.083 per $\mathrm{hr}$ & 4.2 sec. \\
\hline Zandi, et al. [38] & $\begin{array}{l}\text { Regularity, energy \& com- } \\
\text { bined seizure indices }\end{array}$ & $\begin{array}{l}\text { Cumulative Sum } \\
\text { (CUSUM) thresholding }\end{array}$ & NA & $91.00 \%$ & 0.33 per $\mathrm{hr}$ & $9 \mathrm{sec}$. \\
\hline Altaf, et al. [39] & Digital hysteresis & $\begin{array}{l}\text { Linear Support Vector } \\
\text { Machine (LSVM) }\end{array}$ & NA & $95.70 \%$ & $98.00 \%$ & $1 \mathrm{sec}$. \\
\hline Vidyaratne, et al. [40] & $\begin{array}{l}\text { Fractal dimension, spa- } \\
\text { tial/temporal features }\end{array}$ & $\begin{array}{l}\text { Relevance Vector Ma- } \\
\text { chine (RVM) }\end{array}$ & $99.80 \%$ & $96.00 \%$ & 0.1 per $\mathrm{hr}$ & $1.89 \mathrm{sec}$. \\
\hline Sayeed, et al. [16] & Hyper-synchronous pulses & $\begin{array}{l}\text { Signal Rejection Algo- } \\
\text { rithm (SRA) }\end{array}$ & NA & $96.90 \%$ & $97.50 \%$ & $3.6 \mathrm{sec}$. \\
\hline Olokodana et al. [35] & $\begin{array}{l}\text { Petrosian fractal dimen- } \\
\text { sion }\end{array}$ & 1D Kriging Model & $100.00 \%$ & $100.00 \%$ & $100.00 \%$ & $0.85 \mathrm{sec}$ \\
\hline Current Paper & $\begin{array}{l}\text { Fractal dimension, Hjorth } \\
\text { complexity \& Entropy }\end{array}$ & 3D Kriging Model & $97.50 \%$ & $94.74 \%$ & $100.00 \%$ & 0.81 sec. \\
\hline
\end{tabular}

integration of some advanced security features to our proposed medical device as it is IoMT-enabled and can be part of large scale Internet-of-Everything (IoE) or healthcare CyberPhysical Systems (H-CPS) [26], [42].

\section{REFERENCES}

[1] H. M. De Boer, M. Mula, and J. W. Sander, "The global burden and stigma of epilepsy," Epilepsy \& behavior, vol. 12, no. 4, pp. 540-546, 2008.

[2] H. W. Shin, V. Jewells, E. Hadar, T. Fisher, and A. Hinn, "Review of epilepsy-etiology, diagnostic evaluation and treatment," Int J. Neurorehab., vol. 1, no. 130, pp. 2376-0281, 2014.

[3] O. Devinsky, "Sudden, unexpected death in epilepsy," New England J. Med., vol. 365, no. 19, pp. 1801-1811, 2011.

[4] M. J. Pyrcz and C. V. Deutsch, Geostatistical reservoir modeling. Oxford university press, 2014.

[5] J. Wang, Z. Zhang, B. Li, S. Lee, and R. S. Sherratt, "An enhanced fall detection system for elderly person monitoring using consumer home networks," IEEE Trans. Consum. Electron., vol. 60, no. 1, pp. 23-29, 2014.

[6] L.-H. Wang, Y.-M. Hsiao, X.-Q. Xie, and S.-Y. Lee, "An outdoor intelligent healthcare monitoring device for the elderly," IEEE Trans. Consum. Electron., vol. 62, no. 2, pp. 128-135, 2016.

[7] S.-Y. Lee, C. Tsou, and P.-W. Huang, "Ultra-High-Frequency RadioFrequency-Identification Baseband Processor Design for Bio-signal Acquisition and Wireless Transmission in Healthcare System," IEEE Trans. Consum. Electron., vol. 66, no. 1, pp. 77-86, 2020.

[8] J. W. Kim, J. H. Lim, S. M. Moon, and B. Jang, "Collecting Health Lifelog Data From Smartwatch Users in a Privacy-Preserving Manner," IEEE Trans. Consum. Electron., vol. 65, no. 3, pp. 369-378, 2019.

[9] L. Rachakonda, S. P. Mohanty, and E. Kougianos, "iLog: an intelligent device for automatic food intake monitoring and stress detection in the IoMT," IEEE Trans. Consum. Electron., vol. 66, no. 2, pp. 115-124, 2020.

[10] L. Rachakonda, S. P. Mohanty, E. Kougianos, and P. Sundaravadivel, "Stress-Lysis: A DNN-Integrated Edge Device for Stress Level Detection in the IoMT," IEEE Trans. Consum. Electron., vol. 65, no. 4, pp. 474 483, 2019.

[11] S.-Y. Lee, P.-W. Huang, M.-C. Liang, J.-H. Hong, and J.-Y. Chen, "Development of an arrhythmia monitoring system and human study," IEEE Trans. Consum. Electron., vol. 64, no. 4, pp. 442-451, 2018.

[12] N. Dey, A. S. Ashour, F. Shi, S. J. Fong, and R. S. Sherratt, "Developing residential wireless sensor networks for ECG healthcare monitoring," IEEE Trans. Consum. Electron., vol. 63, no. 4, pp. 442-449, 2017.

[13] S. Puranik and A. Morales, "Heart Rate Estimation of PPG Signals with Simultaneous Accelerometry Using Adaptive Neural Network Filtering," IEEE Trans. Consum. Electron., vol. 66, no. 1, pp. 69-76, 2020.
[14] M. A. Sayeed, S. P. Mohanty, E. Kougianos, and H. P. Zaveri, "NeuroDetect: A Machine Learning-Based Fast and Accurate Seizure Detection System in the IoMT," IEEE Trans. Consum. Electron., vol. 65, no. 3, pp. 359-368, 2019.

[15] S. Raj and K. C. Ray, "A personalized point-of-care platform for realtime ECG monitoring," IEEE Trans. Consum. Electron., vol. 64, no. 4, pp. 452-460, 2018.

[16] M. A. Sayeed, S. P. Mohanty, E. Kougianos, and H. P. Zaveri, "eSeiz: An Edge-Device for Accurate Seizure Detection for Smart Healthcare," IEEE Trans. Consum. Electron., vol. 65, no. 3, pp. 379-387, August 2019.

[17] A. Subasi and M. I. Gursoy, "EEG signal classification using PCA, ICA, LDA and support vector machines," Expert Syst. With App., vol. 37, no. 12, pp. 8659-8666, December 2010.

[18] V. P. Nigam and D. Graupe, "A neural-network-based detection of epilepsy," Neurological Res., vol. 26, no. 1, pp. 55-60, January 2004.

[19] S. Supriya, S. Siuly, H. Wang, J. Cao, and Y. Zhang, "Weighted visibility graph with complex network features in the detection of epilepsy," IEEE Access, vol. 4, pp. 6554-6566, September 2016.

[20] M. Behnam and H. Pourghassem, "Singular Lorenz Measures Method for seizure detection using $\kappa \mathrm{NN}$-Scatter Search optimization algorithm," in Proc. of the Sig Processing and Int. Syst. Conf. (SPIS), 2015, pp. 6772

[21] A. Marquez, M. Dunn, J. Ciriaco, and F. Farahmand, "iSeiz: A low-cost real-time seizure detection system utilizing cloud computing," in Proc. IEEE Glob. Hum. Tech. Conf., 2017, pp. 1-7.

[22] P. M. Vergara, E. de la Cal, J. R. Villar, V. M. González, and J. Sedano, "An IoT platform for epilepsy monitoring and supervising," J. Sensors, vol. 2017, July 2017.

[23] S. Faul, G. Gregorcic, G. Boylan, W. Marnane, G. Lightbody, and S. Connolly, "Gaussian process modeling of EEG for the detection of neonatal seizures," IEEE Trans. Biomed. Eng., vol. 54, no. 12, pp. 21512162, 2007.

[24] I. L. Olokodana, S. P. Mohanty, and E. Kougianos, "Krig-Detect: Exploring alternative Kriging methods for real-time seizure detection from EEG signals," in Proc. IEEE 6th World Forum on Internet of Things (WF-IoT), 2020, pp. 1-6.

[25] R. G. Andrzejak, K. Lehnertz, F. Mormann, C. Rieke, P. David, and C. E. Elger, "Indications of nonlinear deterministic and finite-dimensional structures in time series of brain electrical activity: Dependence on recording region and brain state," Physical Review E, vol. 64, no. 6, p. 061907, November 2001.

[26] S. P. Mohanty, V. P. Yanambaka, E. Kougianos, and D. Puthal, "PUFchain: A Hardware-Assisted Blockchain for Sustainable Simultaneous Device and Data Security in the Internet of Everything (IoE)," IEEE Cons. Elect. Mag., vol. 9, no. 2, pp. 8-16, Mar 2020.

[27] T. Radüntz and B. Meffert, "Cross-Modality Matching for Evaluating User Experience of Emerging Mobile EEG Technology," IEEE Trans. Human-Mach. Syst., vol. 50, no. 4, pp. 298-305, 2020. 
[28] W. Shi, J. Cao, Q. Zhang, Y. Li, and L. Xu, "Edge Computing: Vision and Challenges," IEEE Internet Things J., vol. 3, no. 5, pp. 637-646, October 2016

[29] M. Zaleshina and A. Zaleshin, "The brain as a multi-layered map. Scales and reference points for pattern recognition in neuroimaging," European J. of Geog., vol. 8, no. 1, pp. 6-31, February 2017.

[30] E. I. Moser, Y. Roudi, M. P. Witter, C. Kentros, T. Bonhoeffer, and M.-B. Moser, "Grid cells and cortical representation," Nature Rev. Neuroscience, vol. 15 , no. 7, p. 466, 2014

[31] M. S. Caywood, D. M. Roberts, J. B. Colombe, H. S. Greenwald, and M. Z. Weiland, "Gaussian Process Regression for predictive but interpretable machine learning models: An example of predicting mental workload across tasks," Frontiers in Human Neuroscience, vol. 10, p. 647, January 2017.

[32] N. Cressie, "The Origins of Kriging," Math. Geology, vol. 22, no. 3, pp. 239-252, Apr 1990

[33] S. P. Mohanty, Nanoelectronic mixed-signal system design. McGrawHill Education New York, 2015, no. 0071825711.

[34] M. J. Pyrcz and C. V. Deutsch, Geostatistical Reservoir Modeling. Oxford university press, 2014.

[35] I. L. Olokodana, S. P. Mohanty, and E. Kougianos, "Ordinary-kriging based real-time seizure detection in an edge computing paradigm," in Proc. IEEE Int. Conf. Cons. Elect., 2020, pp. 1-6.

[36] A. Géron, Hands-on machine learning with Scikit-Learn and TensorFlow: concepts, tools, and techniques to build intelligent systems. O’Reilly Media, Inc.”, March 2017.

[37] A. H. Shoeb and J. V. Guttag, "Application of machine learning to epileptic seizure detection," in Proc. of the 27th Int. Conf. on Mach. Learn. (ICML-10), 2010, pp. 975-982.

[38] A. S. Zandi, G. A. Dumont, M. Javidan, and R. Tafreshi, "Detection of epileptic seizures in scalp electroencephalogram: an automated real-time wavelet-based approach," J. Clini. Neurophysiology, vol. 29, no. 1, pp. $1-16,2012$

[39] M. A. B. Altaf, C. Zhang, and J. Yoo, "A 16-channel patient-specific seizure onset and termination detection $\mathrm{SoC}$ with impedance-adaptive transcranial electrical stimulator," IEEE J. Solid-State Circuits, vol. 50, no. 11, pp. 2728-2740, 2015.

[40] L. S. Vidyaratne and K. M. Iftekharuddin, "Real-time epileptic seizure detection using EEG," IEEE Trans. Neural Syst. Rehabil. Eng., vol. 25, no. 11, pp. 2146-2156, 2017.

[41] M. A. Sayeed, S. P. Mohanty, E. Kougianos, and H. Zaveri, "iDDS: An edge-device in iomt for automatic seizure control using on-time drug delivery," in Proc. IEEE Int. Conf. Cons. Elect., 2020, pp. 1-6.

[42] V. P. Yanambaka, S. P. Mohanty, E. Kougianos, and D. Puthal, "PMsec: Physical Unclonable Function-Based Robust and Lightweight Authentication in the Internet of Medical Things," IEEE Trans. Consum. Electron., vol. 65, no. 3, pp. 388-397, Aug 2019.

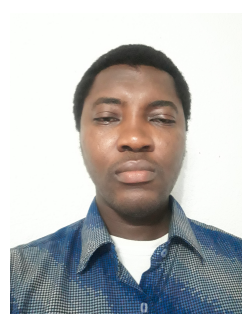

Ibrahim L. Olokodana (M'18) earned his Bachelors of Science (B.Sc.) in Electronic and Electrical Engineering from Obafemi Awolowo University, IleIfe, Nigeria, in 2010 and his Masters of Science (MS) in Electrical and Computer Engineering from Prairie View A \& M University, Texas, in 2017. He received his Ph.D. degree in Computer Science and Engineering at the University of North Texas, Denton, TX at the Smart Electronics Systems Laboratory (SESL). His research interests include the Internet of Things (IoT), sensor design and modeling, smart healthcare, Biomedical Signal Processing, Artificial Intelligence and Brain Computer Interface (BCI).

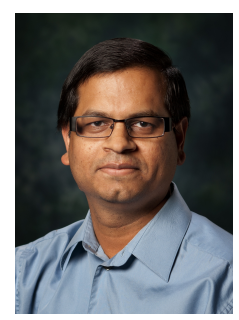

Saraju P. Mohanty (SM'08) received the bachelor's degree (Honors) in electrical engineering from the Orissa University of Agriculture and Technology, Bhubaneswar, in 1995, the masters degree in Systems Science and Automation from the Indian Institute of Science, Bengaluru, in 1999, and the Ph.D. degree in Computer Science and Engineering from the University of South Florida, Tampa, in 2003. He is a Professor with the University of North Texas. His research is in "Smart Electronic Systems" which has been funded by National Science Foundations (NSF), Semiconductor Research Corporation (SRC), U.S. Air Force, IUSSTF, and Mission Innovation. He has authored 350 research articles, 4 books, and invented 4 granted and 3 pending patents. His Google Scholar h-index is 41 and i10-index is 155 with 7200 citations. He is a recipient of 13 best paper awards, Fulbright Specialist Award in 2020, IEEE Consumer Electronics Society Outstanding Service Award in 2020, the IEEE-CS-TCVLSI Distinguished Leadership Award in 2018, and the PROSE Award for Best Textbook in Physical Sciences and Mathematics category in 2016. He has delivered 10 keynotes and served on 11 panels at various International Conferences. $\mathrm{He}$ is the Editor-in-Chief (EiC) of the IEEE Consumer Electronics Magazine (MCE).

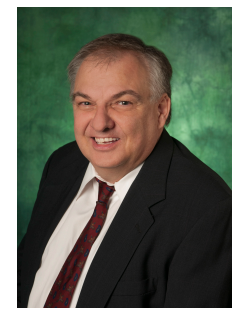

Elias Kougianos (SM'07) received a BSEE from the University of Patras, Greece in 1985 and an MSEE in 1987, an MS in Physics in 1988 and a Ph.D. in EE in 1997, all from Louisiana State University. From 1988 through 1998 he was with Texas Instruments, Inc., in Houston and Dallas, TX. In 1998 he joined Avant! Corp. (now Synopsys) in Phoenix, AZ as a Senior Applications engineer and in 2000 he joined Cadence Design Systems, Inc., in Dallas, TX as a Senior Architect in Analog/Mixed-Signal Custom IC design. He has been at UNT since 2004. He is a Professor in the Department of Electrical Engineering, at the University of North Texas (UNT), Denton, TX. His research interests are in the area of Analog/Mixed-Signal/RF IC design and simulation and in the development of VLSI architectures for multimedia applications. He is an author of over 140 peer-reviewed journal and conference publications.

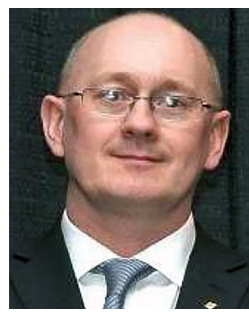

R. Simon Sherratt (F'12) received the B.Eng. degree in Electronic Systems and Control Engineering from Sheffield City Polytechnic, UK in 1992, M.Sc in Data Telecommunications in 1994 and Ph.D. in video signal processing in 1996 from the University of Salford, UK. In 1996, he was appointed as a Lecturer in Electronic Engineering at the University of Reading where he is now Professor of Biosensors. His research topic is signal processing and communications in consumer devices focusing on wearables and healthcare. He is the Emeritus Editor-in-Chief of the IEEE Transactions on Consumer Electronics. 